\title{
Ein empirisches statistisches Verfahren zur Indikation von Datenabnormitäten mittels einer speziellen analytischen Darstellung des Georeliefs
}

\author{
Olga Wälder \\ Technische Universität Dresden, Deutschland
}

\begin{abstract}
Zusammenfassung: Anhand einer Fallstudie aus der Gletscherforschung soll die Anwendung eines empirischen statistischen Verfahrens zur Indikation von Datenabnormitäten mittels einer speziellen analytischen Darstellung von Reliefoberflächen präsentiert werden. Unter bestimmten Annahmen liefert dieses Verfahren zusätzlich ein bequemes Vorhersagemodell zur Gletscherdynamik.
\end{abstract}

\begin{abstract}
An empirical statistical method for the identification of outliers in data sets using a special analytical presentation of relief surfaces is presented. An application of this method for glacial research is shown. Besides, our method gives a comfortable prediction model of the glacier dynamics under some assumptions.
\end{abstract}

Schlüsselwörter: Räumliche Statistik, zeitlicher Trend, Vorhersage, Gletscherdynamik.

\section{Einleitung}

Sowohl die Erfassung als auch die Analyse von räumlich-zeitlichen, glazialen Prozessen gewinnt zunehmend an Bedeutung angesichts des gesteigerten Gefahrenpotentials im Hochgebirgsraum. Das Thema regte zahlreiche methodische und angewandte Studien an, siehe Kaufmann (2004), Kääb et al. (2003), Krainer and Mostler (2004). Die vorliegende Arbeit befasst sich mit einem Teilproblem der Analyse eines Blockgletschers, nämlich mit statistischen Untersuchungen der Genauigkeit der Messungen und mit der Vorhersage von räumlich-zeitlichen Veränderungen der Reliefoberfläche. Das mathematische Werkzeug dieser Analyse stellt eine spezielle analytische Höhendarstellung dar, die sich auf eine unkonventionelle Reliefdarstellung im Amplituden-Frequenz-Bereich stützt, siehe Wälder (2005). An dieser Stelle soll darauf hingewiesen werden, dass diese Darstellung weder eine Spline-Approximation noch eine Wavelet-Transformation des Georeliefs im „,klassischen Sinne“ ist, vgl. Chui (1992).

Im Rahmen einer interdisziplinären Zusammenarbeit mit dem Institut für Geologie und Paläontologie der Universität Innsbruck wurden einige Untersuchungen des Blockgletschers Reichenkar (Tirol) durchgeführt. Die Messergebnisse anhand moderner GPSTechnik bilden dabei eine wichtige Grundlage zur Interpretation der Dynamik aktiver Blockgletscher. Es ist geplant, solche Messungen über einen längeren Zeitraum fortzuführen. Daher ist es wichtig, ein geeignetes statistisches Modell zur Kontrolle der Genauigkeit dieser Messungen und zur Vorhersage der räumlich-zeitlichen Dynamik zu entwickeln. 
Die mathematische Basis der in der vorliegenden Arbeit durchgeführten Untersuchungen stellen spezielle Interpolationsmethoden für ein Datenmaterial dar, das nicht auf konventionelle Weise bearbeitet werden kann, siehe Wälder (2005). Ein geeignetes Beispiel solcher Daten stellen die Untersuchungen am Blockgletscher Reichenkar dar.

Zur Untersuchung des Bewegungsverhaltens dieses aktiven Blockgletschers wurden am Blockgletscher insgesamt 46 markierte Messpunkte (größere Felsblöcke mit eingemeißelten und mit roter Farbe markierten Messmarken) sowie fünf außerhalb des Blockgletschers liegende Fixpunkte vermessen. Die sich ständig in Bewegung befindende Masse überrollt viele solcher Messstellen, dreht sie um, überschüttet sie mit neuen Felsblöcken usw. Man läuft zwar auf dem Gletscher von Jahr zu Jahr näherungsweise identische Routen, Fehler können aber nicht ausgeschlossen werden: man verfehlt beispielsweise einige Messpunkte. Die markierten Messstellen verteilen sich weitgehend nicht gitterförmig auf dem Blockgletscher. Daher war eine geeignete Interpolation der gewonnenen Daten vor Beginn ihrer weiteren Analyse notwendig.

Die erste Messung erfolgte im Jahr 1997, es folgten Nachmessungen in den Jahren 1998 und 2000 bis 2004. Aus Luftbildvergleichen konnte für den Zeitraum von 36 Jahren für den Reichenkar-Blockgletscher ein solcher (tangentialer, d.h. auf dem Hang gemessener) Vorstoß der Zunge ermittelt werden, der einer durchschnittlichen jährlichen Bewegungsrate von $0.64 \mathrm{~m}$ entspricht. Die GPS-Messungen zeigten aber eine deutlich höhere Fließgeschwindigkeit. Für die Jahre 2003-2004 ergab sich eine beinahe dreifache Vergrößerung der Länge der tangentialen Verschiebungsvektoren. Hier werden diejenigen Verschiebungsvektoren als tangential bezeichnet, die sich auf die Verschiebung längs des Hanges von über alle Zeitepochen gleichen, markierten Blöcken beziehen.

Für die zur $(X, Y)$-Ebene senkrechte Absenkung der Blockgletscheroberfläche konnte mittels einer statistischen Untersuchung für diesen Zeitraum zudem eine ungewöhnliche Beschleunigung festgestellt werden, der in der vorliegenden Arbeit näher nachgegangen wird.

Nun stellte sich die Frage, ob dies die Folge der Datenverschmutzung sein konnte oder ob man von einer qualitativen Änderung des zugrunde gelegten Modells sprechen sollte. Die Beantwortung beider Fragen erforderte räumlich-zeitliche statistische Untersuchungen des Datenmaterials, die in den weiteren Abschnitten beschrieben werden.

\section{Modellierung}

Die Untersuchungen des Verschmutzungsgrades von Daten gehören zum Kompetenzbereich der Ausreißerstatistik. Zu einer der ersten Arbeiten, die sich mit Ausreißern bei Zeitreihen beschäftigte, gehört Fox (1972). Im allgemeinen versucht man den Einfluss von Ausreißern zu unterdrücken, weil diese jede Datenanalyse drastisch beeinflussen können. Wir wollen in vorliegender Arbeit bewusst vom Begriff „Ausreißer“ absehen und nur von ,,Datenabnormitäten“ sprechen. Der Grund dafür liegt darin, dass wegen dem spezifischen, Praxis bezogenen Datenmaterial eine strenge theoretische Herleitung sowohl der statistischen Schätzer als auch der Verteilung von Testgrößen fast unmöglich ist: Sieben zeitliche Epochen geben keinen Anlass zu asymptotischen Abhandlungen, der räumlichzeitliche Bezug erfordert die Einführung von mehrdimensionalen Testgrößen usw. 
Hier erweisen sich daher einige empirische Verfahren der räumlichen Statistik bei der oben beschriebenen Problematik als sehr nützlich, siehe Wälder (1999). Nun soll dem konkreten Modellaufbau in Bezug auf seine praktische Anwendung nachgegangen werden.

\subsection{Die Ableitung der analytischen Gleichung des Georeliefs: Trendmodellierung}

Zunächst sollte das räumliche Datenmaterial - die Reliefhöhen in [m] - über verschiedene zeitliche Epochen sozusagen auf ,,einen gemeinsamen Nenner“" gebracht werden. D.h. zeitlich verschiedene, spärliche Messungen von den sich in ständiger Bewegung befindenden Felsblöcken werden auf ein zeitunabhängiges Punktgitter interpoliert. Das grobe Gitter von $5 \times 5$ Punkten entstand auf konventionelle Art und Weise durch die Mittelwertbildung über lokale Bereiche um die vorgesehenen Stützpunkte. Im nächsten Schritt wurde eine sequentielle Reliefinterpolation der Tiefe 3 (Anzahl $p$ der verwendeten Rekursionen) mit zickzack-förmigen Basis-Funktionen nach (1) durchgeführt, um die Visualisierung feinerer Gitterstrukturen zu unterstützen, siehe (Wälder, 2005). Somit konnten analytische Gleichungen des untersuchten Georeliefs für jede zeitliche Epoche ermittelt werden.

$$
\begin{aligned}
S^{p}(x, y)=A & +a_{0} f_{0}(x)+b_{0} f_{0}(y)+c_{0} f_{0}(x) f_{0}(y) \\
& +a_{1} f_{1}(x)+b_{1} f_{1}(y)+c_{1} f_{1}(x) f_{1}(y)+\cdots \\
& \left.+a_{p-1} f_{p-1}(x)+b_{p-1} f_{p-1}(y)\right)+c_{p-1} f_{p-1}(x) f_{p-1}(y)
\end{aligned}
$$

mit den Konstanten $A, a_{k}, b_{k}, c_{k} \in \mathbb{R}, k=0, \ldots, p-1$, wobei

$$
f_{k}(t)= \begin{cases}2^{k} t^{*}, & t^{*} \in\left[0,(1 / 2)^{k}\right] \\ 2^{k}\left((1 / 2)^{k-1}-t^{*}\right), & t^{*} \in\left[(1 / 2)^{k},(1 / 2)^{k-1}+(1 / 2)^{k}\right] \\ 2^{k}\left(t^{*}-(1 / 2)^{k-2}\right), & t^{*} \in\left[(1 / 2)^{k-1}+(1 / 2)^{k},(1 / 2)^{k-2}\right]\end{cases}
$$

$\operatorname{mit} t^{*}=\bmod _{(1 / 2)^{k-2}}(t), k=0,1,2, \ldots$, und

$$
\begin{aligned}
& x=\{\max (x)-\min (x)\} t+\min (x), \\
& y=\{\max (y)-\min (y)\} t+\min (y) .
\end{aligned}
$$

Die Koeffizienten $A, a_{k}, b_{k}, c_{k}$, mit $k=0,1, \ldots, p-1$, sind Amplituden, die für jeden lokalen Bereich und für jede zeitliche Epoche sequentiell angepasst werden, siehe Wälder (2005). Ohne Beschränkung der Allgemeinheit und nur zum Zweck der Vereinfachung der Berechnungen ist es sinnvoll, die realen Gitterkoordinaten auf ein $[0,1] \times[0,1] \mathrm{zu}-$ erst zu transformieren, um diese Amplituden zu ermitteln. Die Rücktransformation ist offensichtlich.

Nachdem eine zeitlich-einheitliche, auf dem räumlichen Frequenzbereich festgelegte Darstellung des Datenmaterials erreicht wurde, konnte eine Gegenüberstellung von Amplitudenzeitreihen in jedem der lokalen Bereiche des Georeliefs erfolgen. Es wurde festgestellt, dass man bei den Zeitreihen 1997-2003 näherungsweise von einer linearen Regression ausgehen konnte. Die zusätzlichen Untersuchungen mit einem quadratischen 
bzw. exponentiellen Ansatz brachten keine deutlichen Verbesserungen. Somit wurde die erste Annahme über einen linearen Trend bei diesen Amplitudenzeitreihen getroffen. Für den Trend des Georeliefs $D$ zum Zeitpunkt $T$ ergab sich unmittelbar:

$$
\begin{aligned}
D^{p}(x, y, T)=\hat{A} & +\hat{a}_{0} f_{0}(x)+\hat{b}_{0} f_{0}(y)+\hat{c}_{0} f_{0}(x) f_{0}(y) \\
& +\hat{a}_{1} f_{1}(x)+\hat{b}_{1} f_{1}(y)+\hat{c}_{1} f_{1}(x) f_{1}(y)+\cdots \\
& +\hat{a}_{p-1} f_{p-1}(x)+\hat{b}_{p-1} f_{p-1}(y)+\hat{c}_{p-1} f_{p-1}(x) f_{p-1}(y) .
\end{aligned}
$$

Hier ist $A m p l=P a r 1_{A m p l} \cdot T+\operatorname{Par} 2_{A m p l}$, wobei $A m p l$ die Terme $\hat{A}, \hat{a}_{k}, \hat{b}_{k}, \hat{c}_{k}$ für $k=0, \ldots, p-1$ bezeichnet, und Par 1, Par2 stellen die Parameter der entsprechenden linearen Regressionen für $A, a_{k}, b_{k}, c_{k}$ dar.

Nach der Trendmodellierung erfolgte die stochastische Modellierung der zufälligen Geooberflächen.

\subsection{Ein empirischer Konfidenzbereich für die mittlere Geooberfläche: Modellierung zufälliger Störungen}

Die zweite Annahme bei unserer Modellierung lag in der Erfassung zufälliger Schwankungen des Georeliefs $R$ mittels:

$$
\begin{aligned}
R^{p}(x, y, T) & =\left(\hat{A}+Z_{A}\right) \\
& +\left(\hat{a}_{0}+Z_{a_{0}}\right) f_{0}(x)+\left(\hat{b}_{0}+Z_{b_{0}}\right) f_{0}(y)+\left(\hat{c}_{0}+Z_{c_{0}}\right) f_{0}(x) f_{0}(y) \\
& +\left(\hat{a}_{1}+Z_{a_{1}}\right) f_{1}(x)+\left(\hat{b}_{1}+Z_{b_{1}}\right) f_{1}(y)+\left(\hat{c}_{1}+Z_{c_{1}}\right) f_{1}(x) f_{1}(y)+\ldots \\
& +\left(\hat{a}_{p-1}+Z_{a_{p-1}}\right) f_{p-1}(x)+\left(\hat{b}_{p-1}+Z_{b_{p-1}}\right) f_{p-1}(y) \\
& +\left(\hat{c}_{p-1}+Z_{c_{p-1}}\right) f_{p-1}(x) f_{p-1}(y)
\end{aligned}
$$

wobei die zufälligen Störgrößen $Z_{A}, Z_{a_{k}}, Z_{b_{k}}, Z_{c_{k}}, k=0, \ldots, p-1$ als trend- und voneinander unabhängig sowie normalverteilt mit Mittelwert Null vorausgesetzt wurden. Der Begriff ,,mittlere Geooberfläche“ mit $D=\mathrm{E}(R)$ (vgl. (2), der obere Index ist hier weggelassen) in der Abschnittsüberschrift wurde hier als Analogon zum Begriff ,Mittelwert“ der klassischen Konfidenzintervallschätzung eingeführt.

Als Schätzer der Standardabweichung jeder Störgröße wurde der Mittelwert der absoluten Abweichungen der für jede zeitliche Epoche nach (1) berechneten Amplituden von ihren durch die entsprechende lineare Regression (vgl. (2)) für diese Zeitpunkte errechneten Werten (d.h, ein Genauigkeitsmaß der entsprechenden linearen Regression).

Anschließend wurden 1000 Simulationen des in (3) dargestellten zufälligen Georeliefs (Höhen) durchgeführt und in jedem der lokalen Bereiche der minimale und maximale Wert berechnet. Diese Werte wurden zu zwei Oberflächen zusammengefasst, einer minimalen und einer maximalen Grenzoberfläche. Der Bereich zwischen diesen Oberflächen wurde dann als empirischer Konfidenzbereich für die mittlere Geooberfläche definiert. Dieser Konfidenzbereich kann einerseits zur Vorhersage der zu erwartenden Gletscherdynamik und anderseits zum Test auf Abnormitäten in den Messungen unter den oben eingeführten Annahmen benutzt werden. Die grundlegende Idee hierzu stellt eine räumlichzeitliche Verallgemeinerung des in Wälder (1999) beschriebenen empirischen Verfahrens dar. 
Nun soll der empirische statistische Test auf Abnormitäten in den Geodaten folgendermaßen formuliert werden: Liegen die Messungen in einem konkreten Jahr zu über M\% in dem unter den oben beschriebenen Modellannahmen errechneten Konfidenzbereich, so kann mit der gewissen Sicherheit von M\% davon ausgegangen werden, dass diese Geodaten keine Abnormitäten aufweisen.

Wenn das Gegenteil eintritt, kann von einer Abnormität in den Geodaten gesprochen werden, die sich sowohl auf Verschmutzung als auch auf die Ungültigkeit der obigen Annahmen zurückführen lassen könnte. Zum Beispiel könnte von einer Steigungsänderung (change point) beim linearen Regressionsansatz gesprochen werden. In diesem Fall sollte der lineare Ansatz in (2) ergänzt werden.

\section{Fallstudie: Messungen am Reichenkar}

Der Reichenkar Blockgletscher befindet sich im Inneren Reichenkar. Das Innere Reichenkar liegt ca. 4 km SE von Längenfeld im Ötztal bzw. 3 km S von Gries im Sulztal (westliche Stubaier Alpen).

Der Reichenkar Blockgletscher entwickelte sich aus einem schuttbedeckten Kargletscher, und stellt einen typischen Blockgletscher mit einem Kern aus Gletschereis dar (siehe Wälder et al., 2004). Die Schuttmassen stammen vor allem aus den steilen östlichen Karwänden. Der relativ flache Zungenbereich ist durch zahlreiche transversale Loben charakterisiert. Die Fließbewegung eines Gletschers wird dadurch festgestellt, dass Punkte auf der Gletscheroberfläche gemessen und deren Lage- und Höhenkoordinaten berechnet werden. Bei Wiederholungsmessungen in bestimmten Zeitabständen werden je nach Aufgabenstellung neue Koordinaten und Höhen berechnet. Aus deren zeitlichen Veränderungen folgt das Fließverhalten des Gletschers.

Abbildung 1 zeigt die Längen der tangentialen Verschiebungsvektoren der Messungen am Gletscher in den Jahren 2002 und 2003, Abbildung 2 können die Längen dieser Vektoren in den Jahren 2003 und 2004 entnommen werden. Die in Abbildung 1 dargestellte Tendenz entspricht ungefähr der in früheren Jahren. Man kann der Legende in Abbildung 2 entnehmen, dass sich die Fließgeschwindigkeit in den Jahren 2003 bis 2004 beinahe verdreifacht hat.

Im ersten Schritt wurden die Messungen in einem ausgewählten Teilgebiet nahe des Zungenbereiches des Gletscher (Abbildung 3) auf ein $5 \times 5$ - Gitter interpoliert und die Ableitung von analytischen Darstellungen wie in (1) für jede der sieben zeitlichen Epochen 1997, 1998 bis 2004 durchgeführt, vgl. Abschnitt 2.1.

Abbildung 4 zeigt die analytische Oberfläche für das Jahr 2004. Somit entstanden 16 Teilbereiche mit 10 eigenen, lokal angepassten Amplituden.

Im zweiten Schritt wurden die Parameter von 160 linearen Regressionen ermittelt. Um den Modellansatz auf seine Relevanz zu testen, wurde zuerst mit den Zeitepochen 1997 bis 2003 gearbeitet. Hierzu wurde bei der Anpassung der linearen Regression immer eines der Jahre ausgelassen und durch das in Abschnitten 2.1 und 2.2 beschriebenen Ansatz vorhergesagt (cross validation). Mit dem in Abschnitt 2.2 eingeführten empirischen statistischen Test konnten keine Abnormitäten festgestellt werden. Dabei konnte eine Sicherheit von $M=95 \%$ gewährleistet werden. 


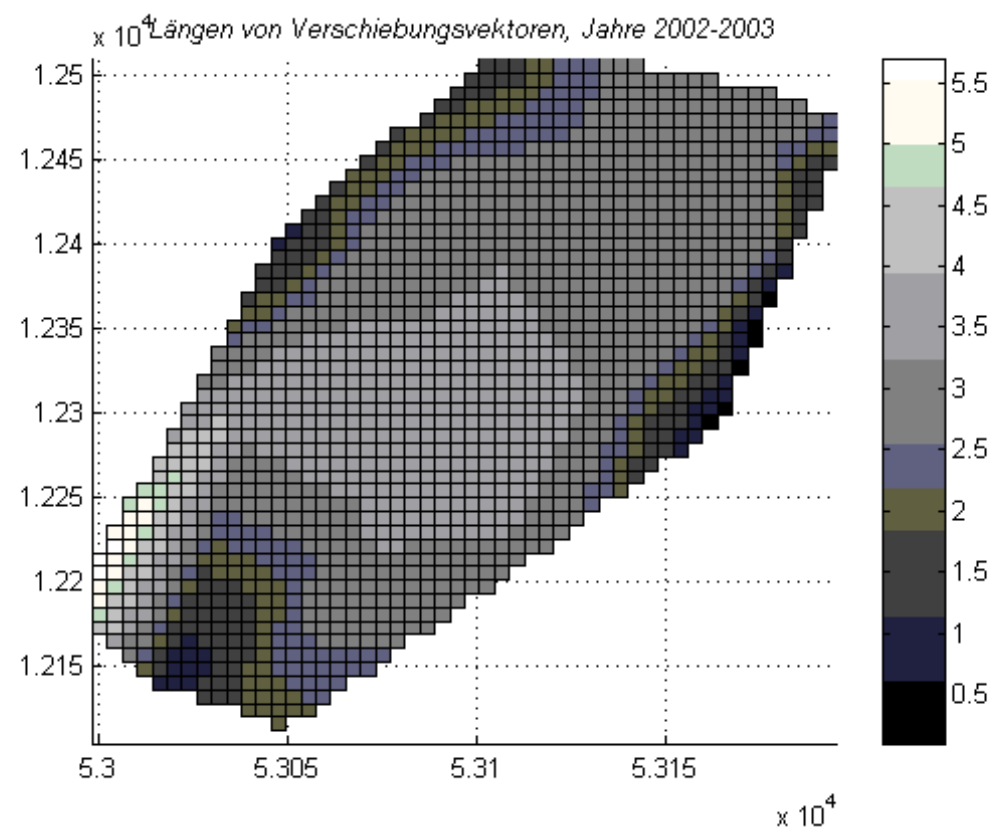

Abbildung 1: Länge der tangentialen Verschiebungsvektoren am Blockgletscher Reichenkar (bikubisch interpoliert in MATLAB) in den Jahren 2002 und 2003, [m].

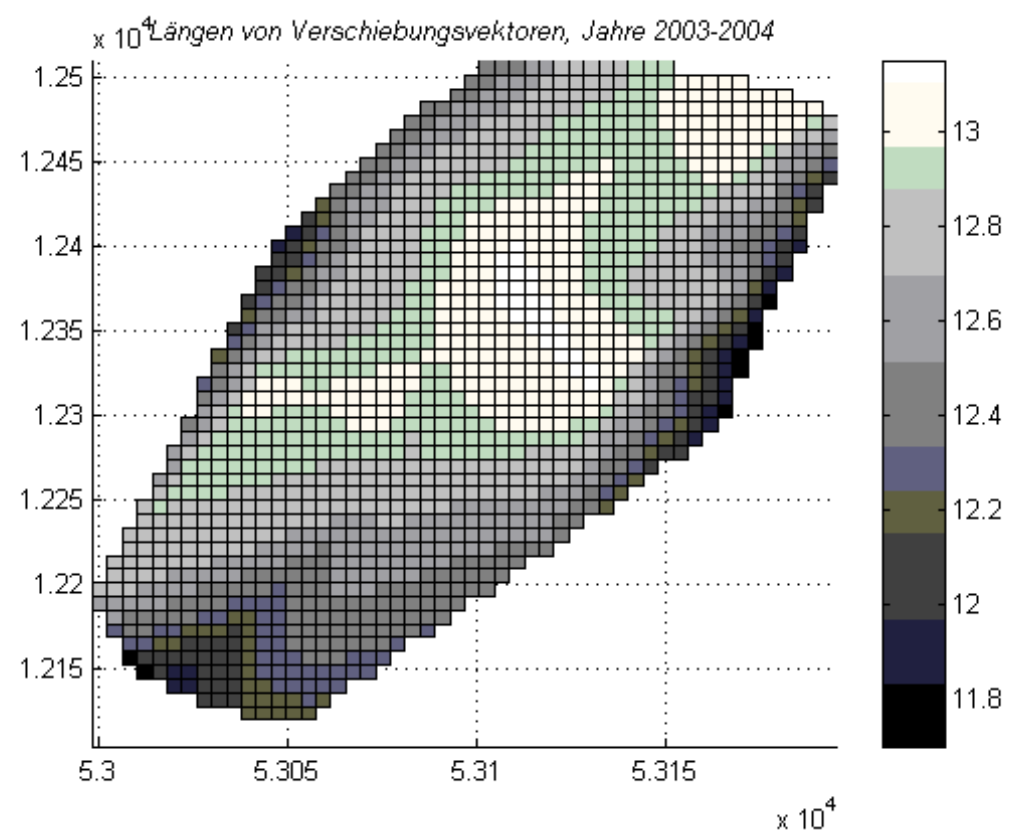

Abbildung 2: Länge der tangentialen Verschiebungsvektoren am Blockgletscher Reichenkar (bikubisch interpoliert in MATLAB) in den Jahren 2003 und 2004, [m]. 


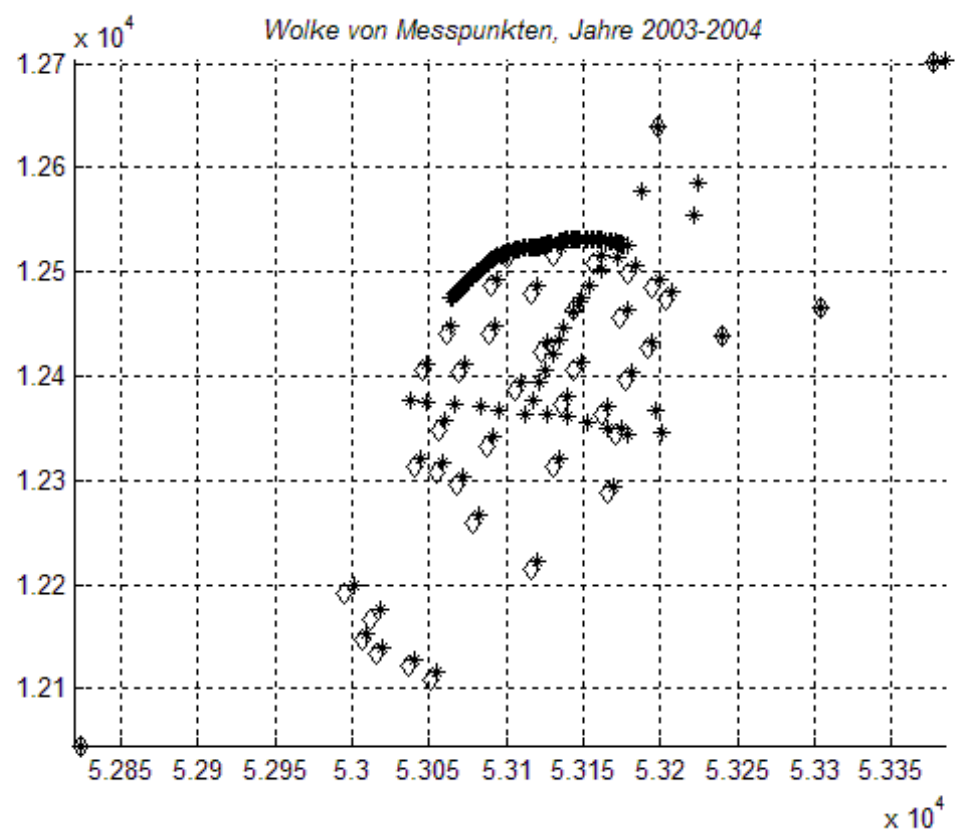

Abbildung 3: Räumlich-zeitliche Darstellung der Messungen am aktiven Blockgletscher Reichenkar (im Jahr 2003 mit Rauten, in 2004 mit Sternen gekennzeichnet). Das Teilgebiet entspricht $[5.305,5.320] \times[1.23,1.25]$ (Koordinaten in $\left.\left[\mathrm{m} \times 10^{4}\right]\right)$.

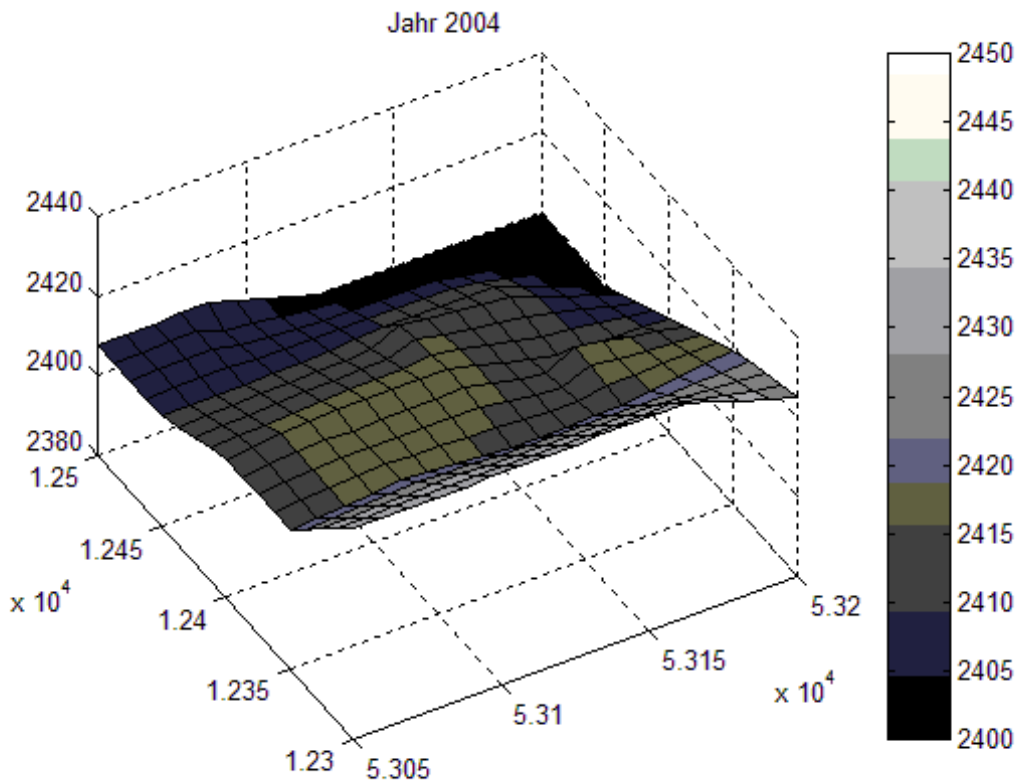

Abbildung 4: Die nach (1) interpolierte Oberfläche für das Jahr 2004, [m]. 


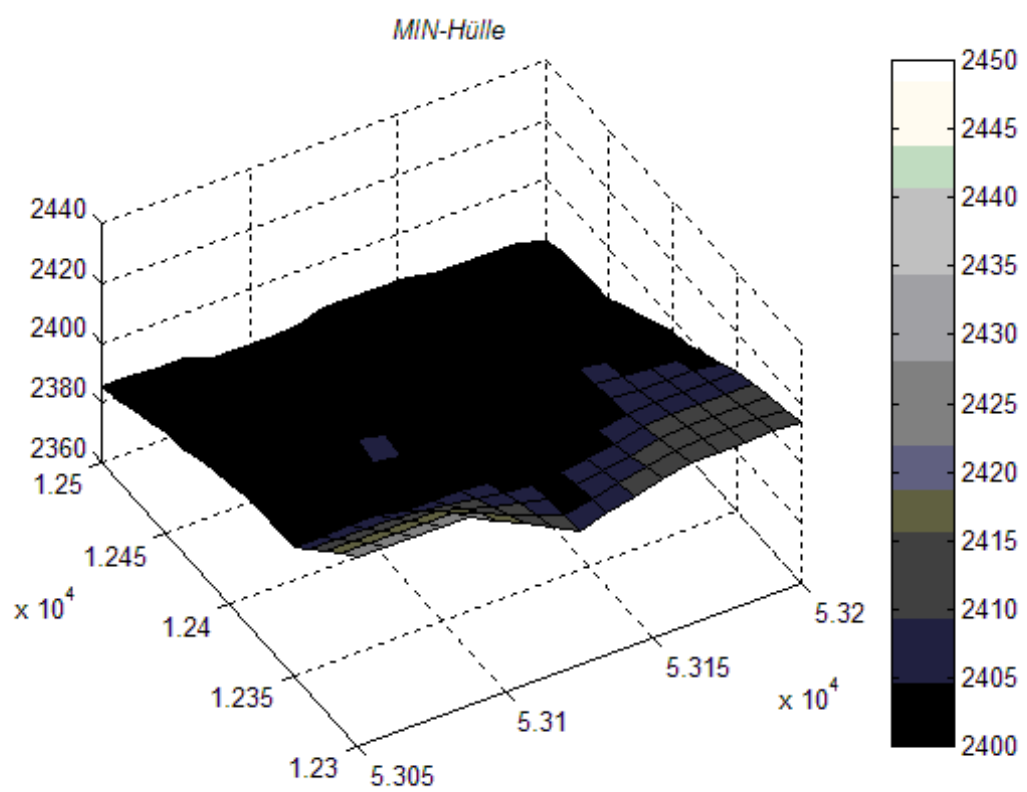

Abbildung 5: Minimale Grenzoberfläche des empirischen Konfidenzbereiches für das Jahr 2004, [m].

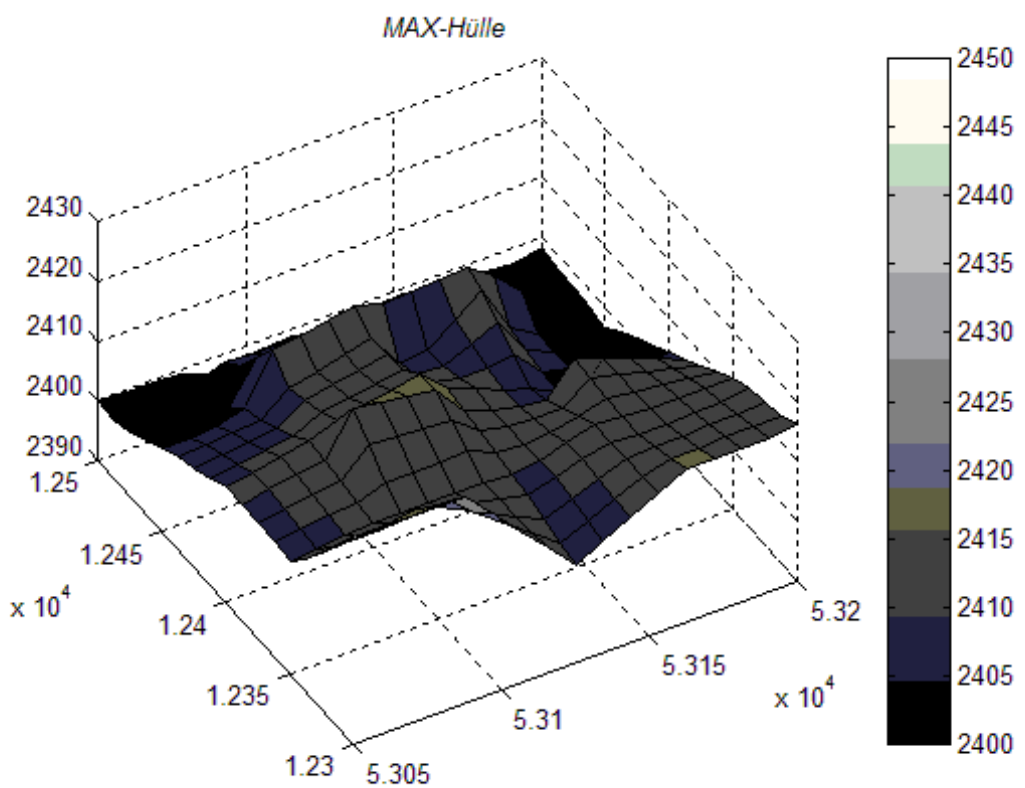

Abbildung 6: Maximale Grenzoberfläche des empirischen Konfidenzbereiches für das Jahr 2004, [m]. 


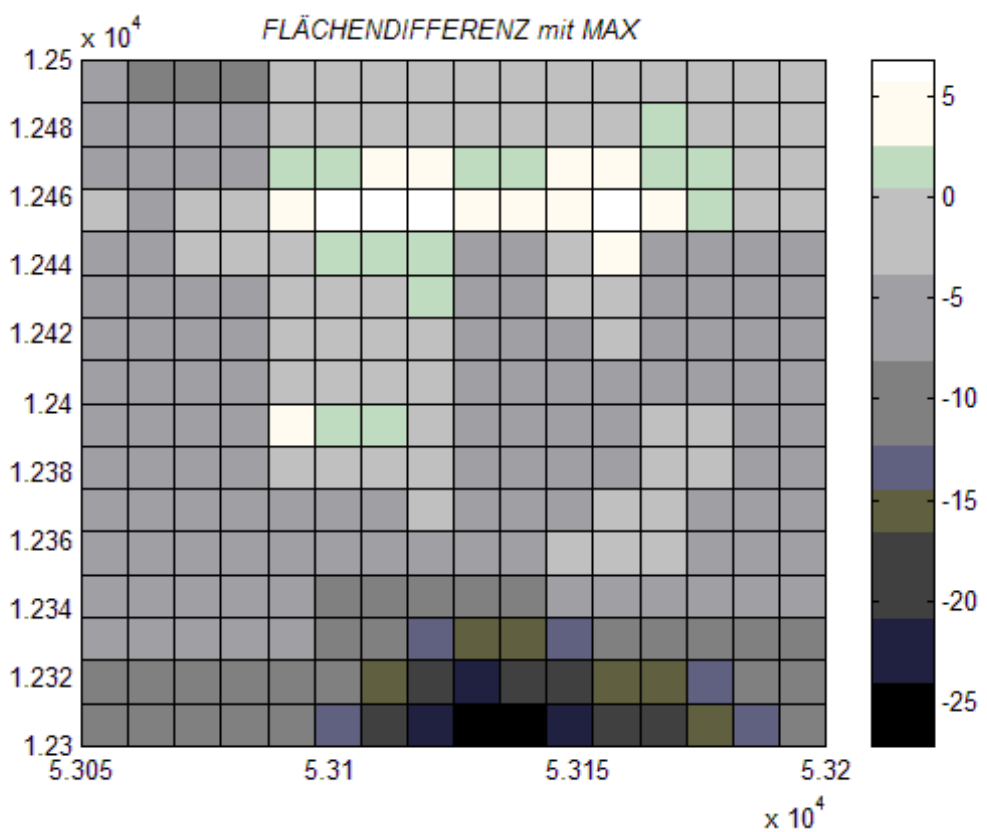

Abbildung 7: Differenz zwischen der maximalen Grenzoberfläche des empirischen Konfidenzbereiches und der Geooberfläche im Jahr 2004, [m].

Nun sollte das Jahr 2004 getestet werden. Mit Hilfe der auf den Amplitudenzeitreihen der Epochen bis einschließlich 2003 basierenden Regressionsansätzen wurden die Schätzungen (Extrapolation) für die entsprechenden Amplituden des Jahres 2004 berechnet. Somit konnte die empirische Vorhersage für das Georelief in dieser zeitlichen Epoche erfolgen. Der Konfidenzbereich für die mittlere Geooberfläche des Jahres 2004 wurde wie in 2.2 ermittelt und besteht aus einer minimalen und maximalen Grenzoberflächen nach 1000 Simulationen, siehe Abbildungen 5 und 6.

Die aus den Höhenmessungen abgeleitete analytische Darstellung für das Jahr 2004 befindet sich zwar stets über der minimalen Grenzoberfläche, die maximale Grenzoberfläche wird aber weitgehend überschritten, siehe Abbildung 7.

Die Korrelation zwischen der vorhergesagten mittleren Geooberfläche und der im Jahr 2004 gemessenen beträgt 0.9. Die Differenz beider Oberflächen kann Abbildung 8 entnommen werden. Die Messungen im Jahr 2004 weisen also einen gewissen Grad an Abnormität auf. Wenn man dabei von einer Datenverschmutzung ausgeht, spricht die Art dieser Differenz eher für einen systematischen Messfehler (ca. $9 \mathrm{~m}$ Überschätzung) in den Daten. Sollte dies der Fall sein, könnten im Datensatz die Höhenmessungen mittels dieser Differenz ,,bereinigt“ und somit plausiblere Aussagen zu den Höhenmessungen erreicht werden.

Die zur Untersuchung benutzten Programme wurden mit Hilfe der Softwaretools DELPHI 7.0 und MATLAB entwickelt. 


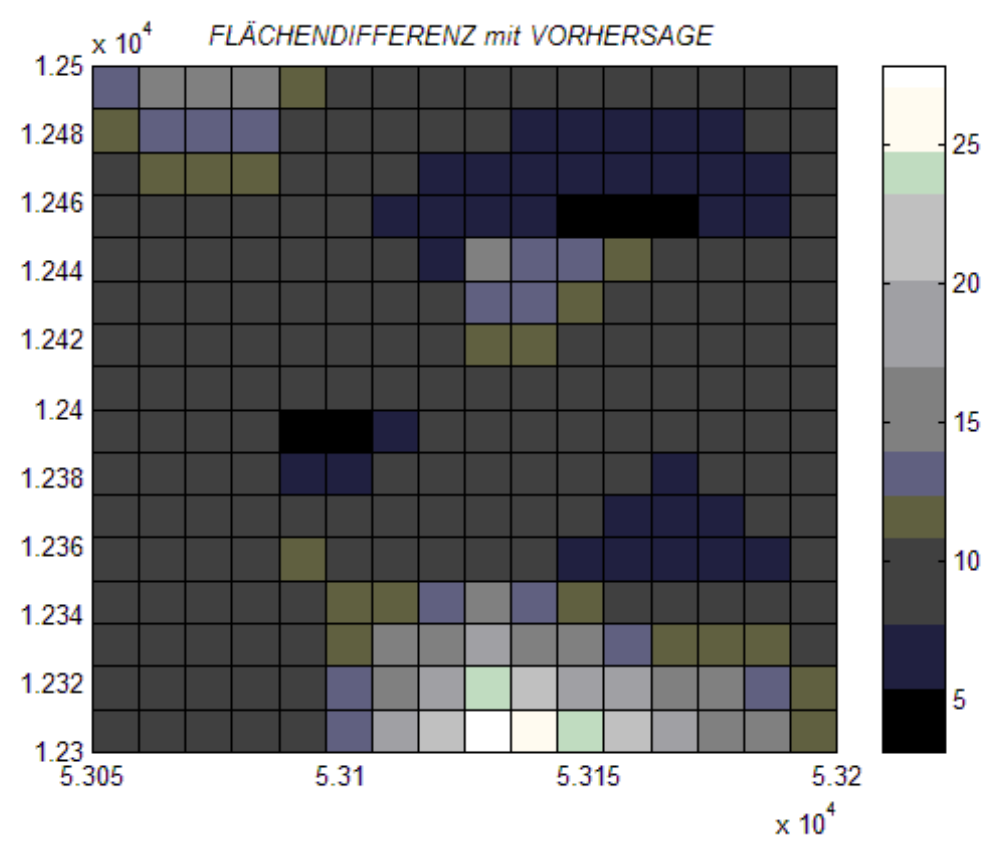

Abbildung 8: Differenz zwischen der im Jahr 2004 gemessenen und der vorhergesagten mittleren Geooberfläche, [m].

\section{Ausblick und Diskussion}

Das in den Abschnitten 2.1 und 2.2 vorgestellte Modell wurde an einem konkreten Anwendungsbeispiel getestet. Die Annahme, dass in den Jahren 1997 bis 2003 keine Abnormitäten auftraten, wurde durch unseren Test bestätigt. Dennoch wurde die Hypothese über Normalität im Jahr 2004 abgelehnt.

Unser Modell stellt eine anwendungsorientierte Ergänzung bzw. eine Verallgemeinerung einiger klassischer Verfahren der räumlichen Statistik dar. Die Trenderfassung durch spezielle, lokal angepasste Oberflächendarstellungen erhöht die Realitätsbezogenheit unseres Verfahrens. Der im Abschnitt 2.2 eingeführte empirische Konfidenzbereich, der sich auf eine wiederholte Simulationen stützt, kann für weitere Anwendungen, insbesondere im Zusammenhang mit zeitlich-räumlichen, zufälligen Modellen, von Interesse sein. In diesem Fall sollten die in Abschnitten 2.1 und 2.2 eingeführten Annahmen Praxis bezogen angepasst bzw. verallgemeinert werden.

Besonders spannend könnte eine spätere Gegenüberstellung von Messungen im Jahr 2005 am Reichenkar und ihrer Vorhersage (Extrapolation) mit dem beschriebenen Ansatz werden: Es wäre durchaus denkbar, dass eine Steigungsänderung bei der linearen Regression der Amplituden bestätigt werden könnte, die ein spezielles Interesse der Gletscherforscher auf sich ziehen dürfte. 


\section{Danksagung}

Die Autorin möchte sich bei Prof. Krainer, Institut für Geologie und Paläontologie der Universität Innsbruck, für die angenehme Zusammenarbeit und für die ihr zur Verfügung gestellten Daten herzlich bedanken. Zudem sei dem Staatsministerium für Wissenschaft und Kunst des Freistaates Sachsen gedankt, das durch die Gewährung einer Habilitationsförderung ebenfalls zu dieser Arbeit beigetragen hat. An dieser Stelle möchte sich die Autorin zudem bei den unbekannten Gutachtern für die hilfreichen und konstruktiven Hinweise bedanken.

\section{Literatur}

Chui, C. K. (1992). An Introduction to Wavelets. Academic Press.

Fox, A. J. (1972). Outliers in time series. Journal of the Royal Statistical Society, Series $B, 43,350-363$.

Kaufmann, V. (2004). Zur Topographie und Morphodynamik des Blockgletschers Hinteres Langtalkar (Schobergruppe, Nationalpark Hohe Tauern, Österreich). Kartographische Nachrichten, 6, 258-261.

Kääb, A., Isakowski, Y., Paul, F., Neumann, A., und Winter, R. (2003). Glaziale und periglaziale Prozesse: Von der statistischen zur dynamischen Visualisierung. Kartographische Nachrichten, 53, 206-212.

Krainer, K., und Mostler, W. (2004). Aufbau und Entstehung des aktiven Blockgletschers im Sulzkar, westliche Stubaier Alpen (Tirol). Geo.Alp, 1, 37-55.

Wälder, O. (1999). Markierungen und Verdünnungen von Punktprozessen. Unpublished doctoral dissertation, Fakultät für Mathematik und Informatik, TU Bergakademie Freiberg.

Wälder, O. (2005). A method for sequential thinning of digital raster terrain models II: mixed locally adaptive wavelet-splines and anisotropy. Photogrammetrie, Fernerkundung, Geoinformation (PFG), 2.

Wälder, O., Krainer, K., und Mostler, W. (2004). Praktische Anwendung von speziellen Spline-Verfahren zur Gletscherkinematik am Beispiel eines aktiven Blockgletschers (Tirol). Österreichische Zeitschrift für Vermessung \& Geoinformation(VGI), 3+4, 107-117.

Adresse des Autors:

Dr. Olga Wälder

Institut für Kartographie

Technische Universität Dresden

Mommsenstrasse 13

D-01062 Dresden

Tel. $+49463 / 36200$

Fax +49 $463 / 37028$

E-mail: Olga.Waelder@mailbox.tu-dresden.de

http://web.tu-dresden.de/kartographie 